\title{
Dinámica anual de peso y condición corporal en vacas de raza Criollo A rgentino
}

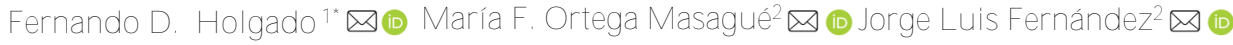 \\ Facultad de Agronomía y Zootecnia. Universidad Nacional de Tucumán, Argentina.
}

\section{Annual dynamics of weight and body condition in Argentine C reole breed cows}

\begin{abstract}
A bstract. Bovine breeding in the Argentine semi-arid Chaco is extensive pastoral and has no or low amount of forage reserves. During the year, the main food is constituted by megathermal pastures characterized by presenting a period of rest and another of active growth in which all the forage is produced. This diet, which varies over time, affects daily weight gains and the body condition of the cows. The objective of this work was to analyze the annual dynamics of weight and body condition of Argentine Creole cows with an exclusively pastoral diet. The information was obtained in the stages of beginning and end of the service, weaning and beginning of the next service in females from 5 to 16 years of age. The statistical model included cow age and year as fixed effects. For the comparison of means, the Tukey-Kramer test was used. Both weight and body condition was affected by age and year. On average, the body condition at the beginning of service was $2.77 \pm 0.38$ points (scale 1 to 5 ) and the weight was $406.8 \pm 25.0$ $\mathrm{kg}$. During the service the weight gain allows to accumulate body reserves in the cows and to reach the weaning stage with a body condition of 3.51 points. From there and until the start of the next service, the cows suffer a loss of body condition in relation to the quality of the forage. In this annual dynamic, females compensate for their weight and can maintain a body condition within the target score $(3 \pm 0.5)$ without compromising the occurrence of fertile heat and achieving high pregnancy rates. However, the mobilization of body reserves has a limit and forage reserves should be available to face very difficult years.
\end{abstract}

Key words: Genetic resources, Cattle breeding, mega thermal pastures, feeding.

Resumen. La cría bovina en el Chaco semiárido argentino es extensiva pastoril y dispone de nula o baja cantidad de reservas forrajeras. Durante el año, el alimento principal está constituido por pasturas megas térmicas caracterizadas por presentar un período de reposo y otro de activo crecimiento en el que se produce la totalidad del forraje. Esta alimentación, variable en el tiempo, afecta las ganancias diarias de peso y la condición corporal de los vientres. El objetivo de este trabajo fue analizar la dinámica anual de peso y condición corporal de vacas Criollo Argentino con una alimentación exclusivamente pastoril. La información fue obtenida en las etapas de inicio y final del servicio, destete e inicio del siguiente servicio en hembras de 5 a 16 años de edad. El modelo estadístico incluyó como efectos fijos la edad de vaca y el año. Para la comparación de medias se empleó el test de Tukey-Kramer. Tanto el peso como la condición corporal fueron afectados por la edad y año. En promedio, la condición corporal al inicio de servicio fue $2.77 \pm 0.38$ puntos (escala 1 a 5 ) y el peso $406.8 \pm 25.0 \mathrm{~kg}$. Durante el servicio la ganancia de peso permite acumular reservas corporales en los vientres y llegar a la etapa de destete con una condición corporal de 3.51 puntos. A partir de allí y hasta el inicio del siguiente servicio las vacas sufren pérdida de condición corporal en relación a la calidad del forraje. En esta dinámica anual, las hembras compensan su peso y pueden mantener una condición corporal dentro del puntaje objetivo $(3 \pm 0.5)$ sin comprometer la ocurrencia de cel os fértiles y lograr al tas tasas de preñez. Sin embargo, la movilización de reservas corporales tiene un límite y se debería contar con reservas forrajeras para hacer frente a años muy difíciles.

Pal abras claves: Recursos genéticos, cría bovina, pasturas megas térmicas, alimentación.

Recibido: 2020-06-10. Aceptado: 2021-03-20

${ }^{1}$ Autor para la correspondencia: fernandodholgado@gmail.com

${ }^{2}$ Instituto de Investigación A nimal del Chaco Semiárido, CIAP, INTA Leales, Tucumán, Argentina. 


\section{Introducción}

El Instituto de Investigación Animal del Chaco Semiárido (IIACS) trabaja en la conservación y caracterización del ganado bovino Criollo Argentino desde el año 1959. A lo largo de estos años la raza ha demostrado un gran desempeño reproductivo, habiendo alcanzado un porcentaje promedio de preñez de $94.5 \%$ en el período 2011 a 2016 (Holgado y Ortega, 2019). Con un intervalo entre partos medio de $370.13 \pm 23.45$ días (Holgado et al., 2019). Para lograr adecuados niveles de eficiencia reproductiva en rodeos de cría, la alimentación del vientre guarda mucha relevancia. No existe un conjunto rígido de reglas y decisiones que puedan ser propuestas para el manejo adecuado de los agroecosistemas, sin embargo, algunos aspectos claves deben ser tenidos en cuenta (Romera et al., 2004). La condición corporal ha sido propuesta como un indicador del estado nutricional y como una herramienta para pronosticar el resultado reproductivo (Correa-Orozco y Uribe-Velásquez, 2010). Su variación durante el ciclo anual, está asociada a la disponibilidad y calidad de los recursos forrajeros y su conocimiento resulta de utilidad para establecer puntos críticos en el manejo del rodeo (Stahringer, 2003). En la región del Chaco Semiárido los sistemas de cría bovina son, en su mayoría, pastoriles y extensivos, con baja o nula disponibilidad de reservas forrajeras y poca utilización de suplementos nutricionales. Los recursos forrajeros predominantes son gramíneas megatérmicas, perennes, naturales y/ o introducidas, de crecimiento estival (Ávila et al., 2014). Estas pasturas poseen una etapa de activo crecimiento (seis meses), ligado al periodo de lluvias y altas temperaturas. En el periodo frío y seco el crecimiento se detiene, por lo tanto la disponibilidad de alimento del invierno depende del forraje reservado en pie (diferido) durante la etapa de crecimiento de las pasturas. Además, teniendo en cuenta la variabilidad de las Iluvias, mes a mes y año a año, la disponibilidad de materia seca acumulada anualmente resulta muy variable. Cuando el rendimiento anual de pasto resulta inferior al esperado para una carga animal determinada, el impacto nutricional en los vientres no se manifestará durante el periodo de crecimiento de la pastura; sino que se traducirá en un volumen menor de reservas en pie para el invierno, generando un déficit cuantitativo que si no es corregido podría afectar la condición corporal del vientre y fertilidad en el próximo servicio. En la bibliografía, numerosos autores han destacado la importancia de que los vientres alcancen, al parto o inicio del servicio, una condición corporal (CC) objetivo para no afectar la fertilidad del rodeo (CorreaOrozco y Uribe-Velásquez, 2010). La oferta de forraje y su cal idad condicionan la ganancia de peso, y el peso y condición corporal de los vientres en diferentes momentos del año. Conocer su dinámica resulta fundamental para determinar estrategias nutricionales y momentos críticos. El objetivo del presente trabajo fue analizar la variación del peso y condición corporal de vientres Criollos a través del ciclo anual, tratar de asociarla con la disponibilidad y calidad de forraje en cada periodo y de generar pautas para mejorar el manejo nutricional de los rodeos y su eficiencia reproductiva. En este trabajo, no se empleó alimentación suplementaria de ningún tipo, con la finalidad de conocer el aporte de las pasturas como único alimento.

\section{M ateriales y M étodos}

La información utilizada en este trabajo corresponde al rodeo de ganado bovino Criollo Argentino existente en el Instituto de Investigación Animal del Chaco Semiárido (IIACS), ubicado en la provincia de Tucumán, Argentina ( $\left.27^{\circ} 11^{\prime} \mathrm{S}, 65^{\circ} 14^{\prime} \mathrm{W}, 335 \mathrm{msnm}\right)$. El clima es subtropical subhúmedo con estación seca, siendo el promedio anual de precipitaciones de $973.3 \pm$ $263.4 \mathrm{~mm}$. El $88.2 \%$ del total de lluvias se concentra en los meses de noviembre a abril. La temperatura media del mes más cálido (enero) es de $25.8^{\circ} \mathrm{C}$, con una máxima media de 32.4 y una mínima media de 19.4 ${ }^{\circ} \mathrm{C}$. La temperatura media más baja corresponde al mes de julio, con un valor de $12.4{ }^{\circ} \mathrm{C}$ y máxima y mínima medias de 20.4 y $4.5^{\circ} \mathrm{C}$ respectivamente. Entre los meses de mayo y septiembre se registran heladas, con un promedio de 16 al año (Holgado y Ortega, 2019). Estas condiciones climáticas permiten que las pasturas megatérmicas tengan un ciclo de crecimiento de aproximadamente seis meses y un periodo de reposo el resto del año. Por lo tanto, la alimentación durante la etapa de reposo se basa en el aprovechamiento de los excedentes de la etapa de crecimiento, reservados en pie (diferidos). La alimentación de los rodeos de cría se basa exclusivamente en el aprovechamiento de las pasturas por pastoreo directo, en verde y diferido, no realizando ningún tipo de suplementación. Las especies forrajeras utilizadas fueron Chloris gayana, M egathyrsus maximus, Brachiaria brizantha y Cynodon dactilon con un rango promedio de producciones acumuladas entre 6000 y $9000 \mathrm{~kg} \mathrm{MS} / \mathrm{ha}$ (Holgado y Ortega, 2019).

El módulo de vacas de cría comprende todos los vientres desde segundo servicio en adelante y ocupa un total de 97 ha en las que se manejan 97 vacas por 
año aproximadamente (carga promedio=1 cabeza/ ha). La categoría de vaquillonas de primer servicio es manejada en forma separada (carga=1.8 cabezas/ ha). El servicio de las hembras, natural a campo, se estaciona en los meses de diciembre, enero y febrero (90 días); asignándose un toro cada 25-35 vacas. El criterio de elección del toro para cada lote está basado en el mínimo parentesco entre reproductores con la finalidad de controlar niveles de consanguinidad en la descendencia. Durante el servicio las vacas son asignadas a los potreros con alta disponibilidad y pastoreos no intensos para brindar la mejor calidad forrajera posible. Finalizado el mismo y hasta el destete, el manejo del sistema prioriza al recurso forrajero, aumentando la carga animal y dando menor posibilidad de selección al animal. La palpación rectal para verificar preñez se realiza a los dos meses de retirados los toros de servicio y se elimina toda vaca que resulte vacía. Al ingreso y salida de servicio y al destete se registra peso y condición corporal (escala de 5 puntos) de los vientres. Los nacimientos ocurren entre el 15/ 9 y 15/12 de cada año, con una concentración del $60 \%$ en el primer mes. Todas las vacas preñadas se manejan en forma conjunta pero, a medida que van pariendo se separar de las preñadas y son asignadas al mejor potrero disponible, buscando mejorar su alimentación. Al nacimiento, los terneros son identificados y pesados. El destete se realiza hacia fines de abril, con una edad aproximada de 205 días. Las prácticas sanitarias en los animales comprenden vacunaciones obligatorias y desparasitaciones de acuerdo a necesidad, siendo los lotes recorridos diariamente por personal capacitado. Los cuidados de los mismos responden a las recomendaciones establecidas en las guías para el cuidado y uso de animales para investigación.

Para este trabajo se empleó información correspondiente a 298 hembras, que ingresaron a servicio entre los años 2011 a 2015. Las categorías consideradas, de acuerdo a la edad de las vacas, fueron: 5, 6, 7-10, 11-12, 13-14 y 15-16 años. No fueron consideradas las categorías vaquillonas y vacas de segundo servicio por ser variables las edades al primer servicio. Se tomaron registros individuales de peso y condición corporal (escala 1 a 5) al inicio y final del servicio, al destete de las crías y al inicio del servicio siguiente. Se empleó un protocolo de trabajo estándar. La condición corporal fue evaluada por dos observadores, previamente capacitados, que realizan esta tarea desde hace 15 años. Además, se evaluó la ganancia diaria de peso como (peso inicial - peso final I duración período), en tres periodos: durante el servicio (90 días), desde la salida de servicio hasta el destete (75 días) y desde destete hasta el servicio siguiente (200 días). Y la ganancia de peso anual (365) desde ingreso a dos servicios consecutivos. Para el análisis de los datos obtenidos se utilizó proc GLM del paquete estadístico SAS. EI modelo incluyó como efectos fijos la edad de la vaca y el año de servicio. También se consideró y evaluó el efecto del momento temporal (inicio y fin de servicio, destetey al momento de inicio del siguiente servicio). Para este último momento de colecta de datos se consideraron los mismos vientres que iniciaron el ciclo anual. Aunque para el inicio del servicio del año siguiente se produce un recambio de animales. Por eso, por ejemplo, la CC4 del año 2013 no coincide exactamente con la CC1 del 2014. Para la comparación de medias se empleó el test de Tukey-Kramer, y un nivel de P menor $5 \%$.

En el cuadro 1 se presenta la influencia de la edad de madre en los pesos y ganancias diarias de peso de vacas de la raza Criollo Argentino, para los distintos momentos de evaluación. En cuanto a los pesos, se observan diferencias significativas en función de la edad de la hembra. En cuanto al peso al inicio del servicio o entore ( $\mathrm{P} 1)$, se observa que los vientres más jóvenes (5-6 años) resultaron más livianas que las de mayor edad. Las de 7-10 años, presentaron un peso intermedio y las vacas de 11 a 16 años los pesos más altos $(P<0.05)$. Estas diferencias tienden a mantenerse para pesos posteriores (P2, P3 y P4). El peso de los vientres no resulta el mejor indicador del estado corporal del animal. Es conocido que el peso corporal del animal está sujeto a variación en función del tamaño y que nivel de reservas. La condición corporal (CC) resulta un mejor predictor del estado nutricional de los mismos, proporcionando un buen índice de grasa subcutánea como fuente de energía disponible (Ayres et al., 2009, Correa-Orozco y Uribe-Velásquez, 2010). Los pesos resultan importantes para conocer la evolución de los mismos a través del tiempo. Es decir, conocer las ganancias de peso de los vientres en diferentes momentos. Las ganancias de peso en diferentes periodos son un reflejo de la alimentación recibida. Cuando las condiciones son favorables, alta disponibilidad y buena calidad, las hembras ganarán peso. Cuando disponibilidad $\mathrm{y} / \mathrm{o}$ calidad son limitantes los vientres movilizarán reservas y acusarán pérdidas de peso. Conocer la dinámica temporal de pesos, ganancias de peso y condición corporal, son fundamentales para inferir como fue la alimentación de los vientres en un determinado lapso de tiempo y establecer pautas para ajustarla en función de objetivos productivos. Coincidentemente con lo mostrado en el cuadro 1, en relación al peso corporal, Arias et al., (1986) señalan que las hembras presentaron un mayor peso en el mes de mayo, antes de entrar al invierno y los menores pesos fueron 
registrados en septiembre al ingresar a servicio. $Y$ que las mayores pérdidas de peso, en vientres de razas Brahman, Hereford y cruzas Brahman-Hereford con servicio de primavera, de registran durante los primeros estadios de lactación (A rias et al ., 1986).

Cuadro 1. Peso corporal $(\mathrm{kg})$ de vacas Criollas de diferentes edades al ingreso (P1) y salida (P2) de servicio, al destete de las crías (P3) y al ingreso a un servicio sucesivo (P4); y aumentos medios diarios (g/ d) durante el servicio (GMP1), desde la salida de servicio hasta el destete (GMP2), desde allí hasta el servicio siguiente (GMP3) e interanual (GMP4).

\begin{tabular}{cccccccccc}
\hline $\begin{array}{l}\text { Edad } \\
\text { (años) }\end{array}$ & $\begin{array}{c}\text { Número } \\
\text { de vacas }\end{array}$ & $\begin{array}{c}\text { P1 } \\
(\mathrm{kg})\end{array}$ & $\begin{array}{c}\text { GMP1 } \\
(\mathrm{kg} / \mathrm{d})\end{array}$ & $\begin{array}{l}\text { P2 } \\
(\mathrm{kg})\end{array}$ & $\begin{array}{c}\text { GMP2 } \\
(\mathrm{kg} / \mathrm{d})\end{array}$ & $\begin{array}{c}\text { P3 } \\
(\mathrm{kg})\end{array}$ & $\begin{array}{c}\text { GMP3 } \\
(\mathrm{kg} / \mathrm{d})\end{array}$ & $\begin{array}{c}\mathrm{P} 4 \\
(\mathrm{~kg})\end{array}$ & $\begin{array}{c}\mathrm{GMP} \\
(\mathrm{kg} / \mathrm{d})\end{array}$ \\
\hline 5 & 75 & $368^{\mathrm{a}}$ & $0.403^{\mathrm{a}}$ & $404^{\mathrm{c}}$ & $0.124^{\mathrm{a}}$ & $411^{\mathrm{c}}$ & $-0.144^{\mathrm{a}}$ & $380^{\mathrm{d}}$ & $0.035^{\mathrm{a}}$ \\
6 & 49 & $380^{\mathrm{a}}$ & $0.433^{\mathrm{a}}$ & $419^{\mathrm{c}}$ & $0.056^{\mathrm{a}}$ & $423^{\mathrm{c}}$ & $-0.144^{\mathrm{a}}$ & $392^{\mathrm{d}}$ & $0.031^{\mathrm{a}}$ \\
$7-10$ & 114 & $414^{\mathrm{b}}$ & $0.422^{\mathrm{a}}$ & $452^{\mathrm{ab}}$ & $0.068^{\mathrm{a}}$ & $456^{\mathrm{ab}}$ & $-0.175^{\mathrm{a}}$ & $419^{\mathrm{bc}}$ & $0.012^{\mathrm{a}}$ \\
$11-12$ & 34 & $438^{\mathrm{c}}$ & $0.388^{\mathrm{a}}$ & $473^{\mathrm{a}}$ & $0.065^{\mathrm{a}}$ & $477^{\mathrm{a}}$ & $-0.169^{\mathrm{a}}$ & $441^{\mathrm{a}}$ & $0.006^{\mathrm{a}}$ \\
$13-14$ & 19 & $423^{\mathrm{b}}$ & $0.398^{\mathrm{a}}$ & $459^{\mathrm{ab}}$ & $0.148^{\mathrm{a}}$ & $468^{\mathrm{a}}$ & $-0.165^{\mathrm{a}}$ & $433^{\mathrm{ab}}$ & $0.025^{\mathrm{a}}$ \\
$15-16$ & 7 & $416^{\mathrm{b}}$ & $0.381^{\mathrm{a}}$ & $450^{\mathrm{b}}$ & $0.004^{\mathrm{a}}$ & $451^{\mathrm{b}}$ & $-0.181^{\mathrm{a}}$ & $412^{\mathrm{c}}$ & $-0.012^{\mathrm{a}}$ \\
\hline
\end{tabular}

a, b, c Diferentes letras en las columnas indican diferencia estadística $(P<0.05)$

En cuanto a las ganancias de peso (cuadro 1), al comparar las diferentes edades de vientres, en diferentes momentos, no se observan diferencias significativas debidas a la edad. Es decir que las ganancias de peso de vientres de diferentes edades fueron similares en todos los períodos evaluados. Esto indica que todas las edades recibieron igual alimentación y respondieron de manera similar.
En el aradro 2 se presentan las variaciones de pesos y ganancias medias diarias en función de los años evaluados. El efecto año fue significativo en ambas variables, reflejando variaciones en el crecimiento debidas a variaciones anuales de las condiciones ambientales, especialmente lluvias, y su influencia en la disponibilidad de forraje.

Cuadro 2. Peso corporal (kg) de vacas Criollas al ingreso (P1) y salida (P2) de servicio, al destete de las crías (P3) y al ingreso a un servicio sucesivo (P4); y aumentos medios diarios (g/ d) durante el servicio (GMP1), desde la salida de servicio hasta el destete (GMP2), desde allí hasta el servicio siguiente (GMP3) e interanual (GMP4) en los años 2011 a 2015.

\begin{tabular}{lcclccccrr}
\hline $\begin{array}{l}\text { Edad } \\
\text { (años) }\end{array}$ & $\begin{array}{c}\text { Número } \\
\text { devacas }\end{array}$ & $\begin{array}{c}\text { P1 } \\
(\mathrm{kg})\end{array}$ & $\begin{array}{c}\text { GMP1 } \\
(\mathrm{kg} / \mathrm{d})\end{array}$ & $\begin{array}{c}\mathrm{P} 2 \\
(\mathrm{~kg})\end{array}$ & $\begin{array}{r}\mathrm{GMP2} \\
(\mathrm{kg} / \mathrm{d})\end{array}$ & $\begin{array}{c}\mathrm{P} 3 \\
(\mathrm{~kg})\end{array}$ & $\begin{array}{r}\mathrm{GMP3} \\
(\mathrm{kg} / \mathrm{d})\end{array}$ & $\begin{array}{r}\mathrm{P} 4 \\
(\mathrm{~kg})\end{array}$ & $\begin{array}{r}\mathrm{GMP4} \\
(\mathrm{kg} / \mathrm{d})\end{array}$ \\
\hline 2011 & 57 & $423^{\mathrm{a}}$ & $0.320^{\mathrm{c}}$ & $452^{\mathrm{a}}$ & $0.158^{\mathrm{a}}$ & $461^{\mathrm{a}}$ & $-0.138^{\mathrm{c}}$ & $431^{\mathrm{a}}$ & $0.023^{\mathrm{b}}$ \\
2012 & 50 & $425^{\mathrm{a}}$ & $0.206^{\mathrm{d}}$ & $444^{\mathrm{ab}}$ & $0.206^{\mathrm{a}}$ & $456^{\mathrm{a}}$ & $-0.292^{\mathrm{d}}$ & $393^{\mathrm{c}}$ & $-0.087^{\mathrm{d}}$ \\
2013 & 55 & $381^{\mathrm{b}}$ & $0.710^{\mathrm{a}}$ & $445^{\mathrm{ab}}$ & $0.050^{\mathrm{b}}$ & $448^{\mathrm{b}}$ & $-0.271^{\mathrm{d}}$ & $389^{\mathrm{c}}$ & $0.023^{\mathrm{b}}$ \\
2014 & 60 & $378^{\mathrm{b}}$ & $0.583^{\mathrm{b}}$ & $430^{\mathrm{b}}$ & $0.058^{\mathrm{b}}$ & $433^{\mathrm{c}}$ & $-0.010^{\mathrm{a}}$ & $431^{\mathrm{a}}$ & $0.147^{\mathrm{a}}$ \\
2015 & 76 & $427^{\mathrm{a}}$ & $0.201^{\mathrm{d}}$ & $446^{\mathrm{a}}$ & $-0.084^{\mathrm{c}}$ & $441^{\mathrm{bc}}$ & $-0.104^{\mathrm{b}}$ & $418^{\mathrm{b}}$ & $-0.025^{\mathrm{c}}$ \\
Media & & 406.8 & 0.404 & 443.4 & 0.078 & 447.8 & -0.163 & 412.4 & 0.016 \\
DE & & 25.0 & 0.231 & 8.1 & 0.112 & 11.3 & 0.118 & 20.3 & 0.086 \\
\hline
\end{tabular}

a, b, c Diferentes letras en las columnas indican diferencia estadística $(P<0.05)$

La mayor ganancia de peso durante el servicio (GMP1), correspondió al año 2013 con 0.710 kg/ d; y la menor ganancia al año 2015 con $0.201 \mathrm{~kg} / \mathrm{d}$, mostrando la magnitud que puede alcanzar el efecto año. Además, se observa que a la mayor ganancia de peso le corresponde un bajo P1 (381 kg) y a la menor ganancia un peso un P1 de $427 \mathrm{~kg}$. Esto estaría indicando que el estado nutricional previo afecta la ganancia de peso de los vientres, como lo expresa Holgado (2002), quien señala que peso o la CC previa de los vientres afecta la ganancia de peso, concluyendo que a mejores estados corporales menores serán las ganancias. Esta tendencia a compensar, también fue observada por Graham, 1982; Ayres et al., 2014) quienes señalan que las vacas con pobre condición corporal al parto pierden menos reservas corporales que se encuentran en mejor estado, pero aun así muestran menor fertilidad (Graham, 1982; Ayres et al., 2014). Holgado et al., (2020b) encuentran una correlación de - 0.59 entre la CC1 y la GMP1, siendo la función lineal resultante GMP1 =1 291 g/ d - 315 CC1. Es decir que los vientres que ingresan con menor $\mathrm{CC}$ al servicio realizan las mayores ganancias de peso, tendiendo así a compensar y achicar las diferencias iniciales. Al analizar y comparar las ganancias de peso del rodeo en su conjunto, en los diferentes momentos evaluados (GMP1, GMP2, GMP3 y GMP4), se observa que las ganancias entre periodos difirieron en forma significativa ( $P<0.05)$, resultando GMP1 $(0.404 \pm 0.231$ $\mathrm{kg} / \mathrm{d})$ ) mayor que GMP2 $(0.078 \pm 0.112 \mathrm{~kg} / \mathrm{d})$ y estas 
dos mayores que GMP3 $(-0.163 \pm 0.118 \mathrm{~kg} / \mathrm{d})$. GMP4 $(0.016 \pm 0.086 \mathrm{~kg} / \mathrm{d})$ representa lo ocurrido en el año y difirió de todas las anteriores.

La ganancia de peso GMP1 $(0.404 \pm 0.231 \mathrm{~kg} / \mathrm{d})$ es importante porque en ese momento se está definiendo el resultado reproductivo y productivo del año. La CC al inicio y la ganancia de peso durante el servicio aportan información al sistema endócrino sobre el estado nutricional del vientre (Correa-Orozco y UribeVelásquez, 2010). Pérez-Clariget, Carriquiry y Soca, 2007, analizan estrategias nutricionales para mejorar la reproducción del ganado bovino y señalan que el flushing, durante los primeros días del servicio, puede resultar favorable para mejorar los índices de procreo. Las GMP1 y GMP2 $(0.078 \pm 0.112 \mathrm{~kg} / \mathrm{d})$, indican que en estos periodos (165 días) los vientres ganan peso $y$, por lo tanto, mejoran su condición corporal. Durante el período invernal (GMP3) de 200 días de duración, los vientres perdieron peso en todos los años evaluados, con un mínimo de $-10 \mathrm{~g} / \mathrm{d}$ en el año 2014 y un máximo de $-0.292 \mathrm{~kg} / \mathrm{d}$ en el año 2012. En general, las pérdidas de peso en invierno se deben tratar de minimizar, para llegar al próximo servicio con una CC objetivo de $3 \pm$ 0.5 puntos. Teniendo en cuenta que durante el invierno el vientre pierde peso y CC, la clave en los planteos de cría de nuestra región sería la de entrar al invierno con una condición corporal de 3.5 puntos, para contar con suficientes reservas corporales para hacer frente a las exigencias energéticas de final de gestación, parto, lactancia y llegar al servicio con condición corporal objetivo $3 \pm 0.5$.

La ganancia de peso a la vuelta del año (GMP4) también fue afectada por el año, mostrando que existen años donde $e l$ vientre tiene ganancias negativas $(-0.087 \mathrm{~kg} / \mathrm{d})$ y pierden peso $(-32 \mathrm{~kg})$, como en 2012. $Y$ años donde tiene ganancias positivas ( 0.147 $\mathrm{kg} / \mathrm{d}$ ) y gana peso (+54 kg en 2014), recomponiendo sus reservas corporales. Sin embargo, para compensar años extremos o rachas desfavorables es aconsejable que los sistemas ganaderos de cría cuenten con reservas forrajeras para apuntalar la alimentación de los vientres en estas situaciones.

Es importante relacionar las ganancias de peso, de los diferentes periodos con la situación de los recursos forrajeros. En este sentido, la magnitud de GMP1 responde muy bien a la situación normal de los meses de Diciembre a Febrero, donde las gramíneas megatérmicas se encuentran en una etapa de activo crecimiento. Esto genera un periodo de alta disponibilidad forrajera y de buena calidad. La calidad del forraje disponible varía en función del estado fenológico de las pasturas y de la intensidad del pastoreo. En el periodo siguiente (GMP2), de 75 días, que va desde fines del servicio (fin de Febrero) al destete (mediados de mayo), los vientres ganan peso $(0.078 \mathrm{~kg} / \mathrm{d})$ en una menor magnitud respecto al periodo anterior. En este periodo la disponibilidad de forraje también es alta, pero los pastoreos empleando cargas más altas y pastoreos más intensos, restringen en cierta medida el nivel de consumo y la calidad de la dieta. Además, es importante tener presente que los vientres han mejorado su estado corporal y que, como lo señala Holgado (2002); la condición corporal inicial presenta una correlación negativa con la ganancia de peso del periodo siguiente. Las GPM 1 y GPM 2, ambas positivas, indican que estos periodos son favorables para que las hembras de cría incrementen el nivel de reservas corporales.

La pérdida de peso (-163.0) que ocurre durante los 200 días que van desde destete (mediados de mayo) hasta inicio del próximo servicio (comienzo de diciembre), representa un escenario nutricional muy diferente a los anteriores y bastante estable año tras año. Ahora, el crecimiento del pasto ha cesado y la disponibilidad de forraje depende de lo reservado durante los 6 meses de crecimiento de la pastura. Este forraje diferido tiene altos niveles de FDN y presenta niveles de 4-5\% PB y 40-50\% digestibilidad (Holgado y Ortega, 2019). Este periodo comprende el último tercio de la gestación, parto e inicio de la lactancia, momento de marcado aumento de los requerimientos de los vientres. En los años donde la cantidad del forraje diferido, acumulado del verano, resulta menor a lo requerido los vientres podrían perder peso más intensamente debido a problemas de disponibilidad, pudiendo alargar el anestro postparto, afectar la aparición de celos fértiles y consecuente tasa de preñez y/ o intervalo entre parto (A rana y Velásquez, 2012).

En el cuadro 3 se presenta el afecto de la edad del vientre sobre la CC en diferentes momentos del año. La CC es un indicador del nivel de reservas corporales y su magnitud al parto $y /$ o inicio del servicio guarda una fuerte relación con el desempeño reproductivo de la hembra bovina y fertilidad del rodeo (Stahringer, 2003, Correa-Orozco y Uribe-Velásquez, 2010). La edad de madre tuvo efecto significativo sobre la CC en todos los momentos evaluados. La CCl, inicio del servicio, es muy importante porque informa al sistema endócrino sobre la situación energética de la hembra. Las vacas más jóvenes (5-6 años) Ilegaron con una menor CCl $(P<0.05)$ que los vientres de mayor edad, indicando la necesidad de prestar mayor atención a estas edades, especialmente en años difíciles. Es de destacar la buena condición de los vientre de 11 a 16 años, mostrando capacidad de mantener un excelente 
estado corporal hasta edades muy avanzadas, justificando la mayor longevidad observada en la raza. Estas diferencias debidas a edad del vientre persistieron después del entore, ya que las ganancias de peso entre edades fueron similares.

Cuadro 4. Condición corporal (CC, escala 1 a 5) de vacas Criollas al ingreso (CC1) y salida (CC2) de servicio, al destete de las crías (CC3) y al ingreso a un servicio gsucesivo (CC4) en los años 2011 a 2015.

\begin{tabular}{lccccc}
\hline Año & $\begin{array}{c}\text { Número de } \\
\text { vacas }\end{array}$ & $\begin{array}{c}\text { Inicio Servicio } \\
\text { CC1 }\end{array}$ & $\begin{array}{c}\text { Fin Servicio } \\
\text { CC2 }\end{array}$ & $\begin{array}{c}\text { Destete } \\
\text { CC3 }\end{array}$ & $\begin{array}{c}\text { Inicio Siguiente } \\
\text { Servicio CC4 }\end{array}$ \\
\hline 2011 & 57 & $2.86^{\mathrm{b}}$ & $3.64^{\mathrm{a}}$ & $3.64^{\mathrm{a}}$ & $3.11^{\mathrm{a}}$ \\
2012 & 50 & $3.08^{\mathrm{a}}$ & $3.40^{\mathrm{b}}$ & $3.54^{\mathrm{b}}$ & $2.69^{\mathrm{b}}$ \\
2013 & 55 & $2.52^{\mathrm{c}}$ & $3.30^{\mathrm{b}}$ & $3.46^{\mathrm{bc}}$ & $2.30^{\mathrm{c}}$ \\
2014 & 60 & $2.24^{\mathrm{d}}$ & $3.17^{\mathrm{c}}$ & $3.49^{\mathrm{bc}}$ & $3.14^{\mathrm{a}}$ \\
2015 & 76 & $3.14^{\mathrm{a}}$ & $3.33^{\mathrm{b}}$ & $3.40^{\mathrm{c}}$ & $2.76^{\mathrm{b}}$ \\
Media & & 2.77 & 3.37 & 3.51 & 2.80 \\
DE & & 0.38 & 0.17 & 0.09 & 0.34 \\
\hline
\end{tabular}

a, b, c Diferentes letras en las columnas indican diferencia estadística $(P<0.05)$

Al analizar y comparar la CC del rodeo entre los diferentes momentos evaluados (CC1, CC2, CC3 y CC4) se observa que la $\mathrm{CC} 1$, al inicio servicio, representa el valor más bajo $(2.77 \pm 0.38)$ difiriendo $(\mathrm{P}$ $<0.05$ ) de CC2 y CC3. No difiere de la CC4 que representa la CC del rodeo al momento de entrada a servicio 1 año después, antes del recambio de animales. Es decir que la CC se mantuvo entre un servicio y otro. La CC de los vientres se eleva durante el servicio para llegar a un valor de $3.37 \pm 0.17$ (CC2) al finalizar el entore. Es decir que durante los 90 días de duración del servicio las hembras ganaron 0.60 puntos de condición corporal, lo cual concuerda con la ganancia de peso de $404 \mathrm{~g} / \mathrm{d}$ registrada en ese periodo. Durante el periodo desde fin de servicio hasta destete (75 días) se observa un incremento moderado de CC de 0.14 puntos. Su magnitud es consistente con la GMP2 $(0.078 \mathrm{~kg} / \mathrm{d})$ lograda en este periodo. Pero lo importante es que los vientres llegan a destete con una CC3 media de $3.51 \pm 0.09$; la cual difiere $(P<0.05)$ de la CC2 (3.37 \pm 0.17$)$. Es decir, con un nivel de reservas corporales adecuado para movilizarlas en función de necesidades futuras. La condición corporal promedio al ingresar al siguiente servicio (CC4) fue $2.80 \pm 0.34$. Es decir, que las vacas registraron una pérdida promedio de 0.71 puntos desde CC3 a CC4, lo que es coherente con la pérdida de peso de $-0.163 \pm 0.118$ $\mathrm{kg} / \mathrm{d}$ observada en ese periodo. Es así que los puntos de condición corporal ganados durante el periodo servicio-destete (0.74) fueron fundamentales para hacer frente a la etapa de forrajes diferidos, especialmente en el último tercio de gestación y primero mes de lactancia, donde se produce una pérdida de 0.71 puntos. Holgado et al., 2020 a, para el mismo rodeo, pero para los años 2013-2016, registraron una pérdida de $C C$ de 0.57 puntos desde el destete (CC3) hasta el ingreso a servicio (CC4). Al descomponer esta disminución de la CC en dos sub períodos: Destete a Parto (165 días) y desde Parto a inicio de servicio (35 días), encuentran que el $63 \%$ (0.36 puntos) ocurre en la etapa de 165 días y que el 37 $\%$ (0.21 puntos) corresponden a la etapa postpartopreservicio. Es decir, que en esta última se produciría un balance energético mucho más intenso, a pesar que el manejo del rodeo contempla la separación de la vaca que va pariendo y su envío al mejor potrero disponible. Generalmente uno reservado para este fin. Esto coincide con lo señalado por Arana y Velásquez (2012), cuando expresan que el periodo post parto es una etapa crítica en el sistema de cría, caracterizada por una pérdida progresiva de CC. Esto debido al incremento agudo del gasto energético en producir leche. Además, el consumo restringido de materia seca lleva a un balance energético marcadamente negativo. Finalmente analizan diferentes alternativas de suplementación de los vientres para compensar estas situaciones. Las variaciones de CC en el tiempo resultan consistentes con las ganancias de peso logradas en cada periodo y reflejan la situación nutricional en cada uno de ellos. La capacidad de utilización de las reservas corporales durante períodos de balance energético negativo es considerada como una habilidad en vacas de cría (A rana y Uribe, 2012); teniendo esta afirmación como límite la necesidad de llegar al inicio del servicio con una CC de $3 \pm 0.5$, ya que el estado nutricional al inicio del servicio tiene estrecha relación con la eficiencia reproductiva del rodeo. De acuerdo a estos resultados, lograr una CC de 3.5 al ingreso al invierno resulta estratégica, porque marca el nivel de reservas con las cuales el vientre ingresará al invierno en condiciones de enfrentar las pérdidas de peso y CC que normalmente ocurren. Es decir, con la cantidad de reservas necesarias para ser movilizadas ya que el consumo de forraje diferido, de baja calidad, no es suficiente para cubrir los requerimientos de los vientres en último tercio gestación, parto y comienzos de la lactancia. 
La CC1, al inicio del servicio, es muy importante ya que brinda información al sistema neuroendocrino sobre el estado nutricional de la hembra. En el cuadro 4 se observa que la $\mathrm{CC} 1$ varió en función del año, entre un mínimo de 2.24 (2014) y un máximo de 3.14 (2015). Sin embargo, los porcentajes de preñez del rodeo no difirieron en función de año, dando un valor promedio de $94.5 \pm 2.7 \%$ (Holgado y Ortega, 2019). Holgado et al., 2019, al analizar la relación entre la CC al parto y al inicio del servicio con el intervalo entre partos (IEP) de los vientres Criollos encuentra que la CC1 influyó en el IEP de los años 2014 y 2015, siendo sus magnitudes $394.7 \pm 24.3$ y $350.2 \pm 29.7$ días respectivamente. Concluyen que existe una correlación negativa -0.39 entre la CC1 y el IEP, es decir que a mejor CC menor IEP. Esto mismo es señalado por A rias et al., 1998, quienes mencionan que una elevada tasa de preñez requiere condiciones corporales iguales o superiores a 4 (escala 1 a 9 puntos) al inicio del servicio. Para este mismo rodeo experimental, Holgado et al., (2019) encontraron que la CC al inicio del servicio presentaba una mayor correlación con el intervalo entre partos (IEP) que la CC al parto. EI IEP se acortó en la medida que la CC aumento (IEP $=426.9$ - 21.3 (C1), el IEP medio fue de $370.1 \pm 23.5$ días, para los 5 periodos evaluados.

El periodo postparto en los planteos de cría bovina es una etapa crítica que se caracteriza por una pérdida progresiva de condición corporal (Montiel y Ahuja, 2005). El incremento en el gasto energético de la hembra debido a la producción de leche, unido a un consumo de materia seca de baja calidad, genera un balance negativo. Si al inicio del servicio las hembras poseen pocas reservas corporales, el consumo de forraje estará direccionado a suplir el gasto energético según un orden de importancia. El uso de la energía disponible para el animal tiene prioridades: metabolismo basal, actividad, reservas energéticas, preñez, lactación, reservas energéticas adicionales, ciclo estral-celo-preñez (Short y Adams, 1988). El patrón general del balance energético en un vientre es negativo al inicio del parto, luego se acentúa y llega a un punto mínimo en la primera o segunda semana postparto-lactación. De aquí en adelante se genera un incremento positivo y estable de balance hasta el momento en que un nuevo ciclo reproductivo se inicie (Beam y Butler, 1998; De Vries et al., 1999; Berry et al., 2006).

Este patrón se repite anual mente, presentando los vientres un nivel mínimo de reservas corporales al inicio del servicio. Estas se incrementan hasta el destete $y$, a partir de ese momento comienzan a declinar. Lo que es muy importante controlar su intensidad, lo cual puede ser crítico cuando la disponibilidad de forraje pasa a ser el factor limitante. En sistemas pastoriles, la producción anual de materia seca es altamente dependiente de las lluvias. $Y$ las Iluvias resultan muy variables entre años. Por lo tanto, para garantizar la estabilidad del sistema de producción es aconsejable contar con estrategias para alcanzar el equilibrio. Una alternativa sería el manejo de reservas forrajeras que compensen años problema. Otra sería el manejo de la carga. Es importante destacar que en los años evaluados en el presente trabajo la disponibilidad de forraje estuvo dentro de lo previsible, no presentando situaciones extremas o rachas desfavorables. Faltas en la disponibilidad de forraje habría acentuado la pérdida de peso desde mayo a diciembre $y$, muy probablemente afectado este equilibrio anual observado.

\section{Conclusiones}

La condición y el peso corporal variaron en función de la edad de los vientres, presentando los animales más jóvenes (5 y 6 años) los menores valores. Esto indica la necesidad de prestar mayor atención a esta faja etaria. Los pesos, ganancias de peso y condición corporal fueron afectados por efecto del año. Se aprecia un incremento de ambas variables desde inicio del servicio (Diciembre) a destete (Mayo). Y, desde Mayo a diciembre se registra una disminución del peso y condición del vientre. Esta dinámica representa un patrón anual que guarda relación con la disponibilidad y calidad de los recursos forrajeros, reflejados en las ganancias de peso de los animales. La condición corporal promedio al inicio del servicio en hembras de raza Criollo Argentino fue de 2.77 puntos y estuvo comprendida dentro del puntaje objetivo ( $3 \pm$ 0.5). Esto es clave para alcanzar altos porcentajes de preñez. En consecuencia, es muy importante que los vientres, desde diciembre a mayo acumulen reservas corporales en cantidad suficiente para hacer frente a la pérdida de condición corporal del último tercio de gestación $y$, fundamentalmente, en el inicio de la lactancia. En este sentido, resulta estratégico que la CC3 (destete) alcance un valor de 3.5 puntos. De esta manera, a pesar de la pérdida de 0.71 puntos de CC en invierno, las vacas logran llegar al servicio con condición corporal dentro del puntaje objetivo, sin comprometer la reproducción. Sin embargo, resulta aconsejable que los sistemas pastoriles cuenten con reservas forrajeras para hacer frente a situaciones nutricionales más desfavorables y permitan reforzar la alimentación invernal cuando la CC3 no sea la adecuada. 


\section{Literatura Citada}

Arana, D.G.; Uribe Velásquez, L.F. 2012. Estrategias para mejorar la condición corporal postparto en vacas de carne. www.scielo.org.co/ scielo.php?scrip=sci_arttexxt\&pi $\mathrm{d}=$ =\$1657-95502012000100008

Arias, A.A.; Ibarra, J.C.; Panario, C.A. y Slobodzian, A. 1986. Crecimiento desde el nacimiento hasta la madurez de hembras Brahman, Hereford y sus cruzas, variaciones de peso estacionales. Rev. Arg. Prod. Anim. Vol 6, N 11-12: 695-706.

Arias A.A., Revidatti, M.A.; Capellari, A. Slobodzian, 1998. Efectos del destete precoz sobre el peso vivo, la condición corporal y la preñez de vientres cruza en Corrientes. Fac de Ciencias Veterinaria. UNNE INTA.

Avila, R.; Barbera, P.; Blanco, L.; Burghi, V.; De Battista, J.P.; Frigerio, K.; Gándara, L.; Goldfarb, M.C.; Griffa, S.; Leal, K.; Kunst, C.; Lacoste, S.M.; Lauric, A.; Calsina, M.; Lean, G.M.; Nenning, F.; Otondo, J.; Petruzzi, H.; Pizzio, R.; Pueyo, J.D.; Ré, A.E.; Ribotta, A.; Romero, L.; Stritzler, N.; Tomas, M.A., Carbonell, C.T.; Ugarte, C.; Veneciano, J. 2014. Gramíneas forrajeras para el subtrópico y el semiárido central de la Argentina. ISBN-978-987521-551-1. Sitio Argentino de Producción A nimal.

Ayres H, Ferreira RM, Torres-Junior JRS, Demetrio CGB, de Lima, Baruselli PS. 2009. Validation of body condition score as a predictor of subcutaneous fat in Nelore (Bos indicus) cows. Livest Sci, 123:175-179. https:/ / doi.org/ 10.1016/ j.livsci.2008.11.004

Ayres $\mathrm{H}$, Ferreira RM, Torres-Junior JRS, Demetrio CGB, Sa Filho MF, Gimenes LU, Penteado L, D'Occhio MJ, Baruselli PS. 2014. Inferences of body energy reserves on conception rate of suckled Zebu beef cows subjected to timed artificial insemination followed by natural mating. Theriogenology 82:529536.

https:/ / doi.org/ 10.1016/ j.theriogenology.2014.04.0 26

Beam, S. W. and W. R. Butler. 1998. Energy balance, metabolic hormones, and early postpartum follicular development in dairy cows fed prilled lipid. J. Dairy Sci. 81:121-131. https:/ / doi.org/ 10.3168/ jds.S0022-0302(98)75559-6

Berry, D. P., R. F. Veerkamp, and P. Dillon. 2006. Phenotypic profiles for body weight, body condition score, energy intake, and energy balance across different parities and concentrate feeding levels. Livest. $\quad$ Sci., 104: 1-12. https:/ / doi.org/ 10.1016/ j.livsci.2006.02.012

Correa-Orozco, A. y L.F. Uribe-Velásquez. 2010. La condición corporal como herramienta para pronosticar el potencial reproductivo en hembras bovinas de carne. Rev. Fac. Nac. Agron. Medellín 63(2): 5607-5619.

DeVries M.J., Van der Beek S., Kaal-Lansbergen LMTE, Ouweltjes W., Wilmink JBM. 1999. Modeling of energia balance in early lactation and the effect of energy deficits in early lactation on first detected estrus postpartum in dairy cows. J. Dairy Sci. 82: 1927-1934. https:/ / doi.org/ 10.3168/ jds.S00220302(99) 75428-7

Graham J. F. 1982. The effect of body condition of beef cows at calving and post calving nutrition on calf growth rate and cow fertility. Proc Aust Soc Anim Prod. 14:309-312.

Holgado, F. D. 2002. Relación de la condición corporal con el peso de entore y la ganancia de peso durante el servicio. Rev. Arg. Prod. Animal Vol. 22, 1:305306.

Holgado F. D. y M. F. Ortega. 2019. Caracterización productiva del bovino Criollo Argentino: periodo 2006-2016. Ediciones INTA, Bs. As. Argentina ISBN 978-987-521-987-8. $26 \mathrm{p}$.

Holgado F. D., R. E. Martínez, G. Cantarela, M.F. Ortega y J.L. Fernández. 2019. Influencia de la condición corporal al parto y al ingreso al servicio sobre el intervalo entre partos en vacas Criollas adultas. Actas Iberoamericanas de Conservación Animal. AICA 14: 60-63.

Holgado F. D., M. F. Ortega, G. Cantarela, y R. E. Martínez. 2020a. Condición corporal de la vaca Criolla en tres momentos: destete, Parto, y servicio. Anais do $\mathrm{XX}$ Simposio Iberoamericano sobre conservación e uso de recursos zoogenéticos locais (Corumba). Documentos 163, EMBRAPA, Brasil 2020, ISSN 1981-7233.

Holgado F.D., Rabasa A., M.F. Ortega, y J.L. Fernández. 2020b. Condición corporal al inicio del servicio y ganancia de peso durante el servicio en vacas Criollas. Anais do XX Simposio Iberoamericano sobre conservación e uso de recursos zoogenéticos locais (Corumba). Documentos 163, EMBRAPA, Brasil 2020, ISSN 1981-7233.

Montie F, Ahuja C, 2005. Body condition and suckling as factors influencing the duration of postpartum anestrus in cattle: a review. Anim Reprod Sci 2005; 85:

$1-26$.

https:/ / doi.org/ 10.1016/ j.anireprosci.2003.11.001

Pérez-Clariget R., M. Carriquiry, y P. Soca. 2007. Estrategias de manejo nutricional para mejorar la reproducción en ganado bovino. Arch. Latinoam. Prod. Anim. 15 (Supl. 1):114-119. https:/ / ojs.alpa.uy/ index.php/ ojs_files/ article/ vie w/ 2726 
Romera, A.J., S. T. Morris, J. Hodgson, W. D. Stirling, S. S. J. R. Woodward. 2004. A model for simulating rule-based management of cow-calf systems. Computers and Electronics in A griculture 42, 67-86.

SAS Institute Inc. 2004. SAS Online 9.1.03 Cary, NC: SAS, Institute Inc.

Short, R. E., and D. C. Adams. 1988. Nutritional and hormonal interrelationships in beef cattle reproduction. Can. J. A nim. Sci., 68: 29-39. https:/ / doi.org/ 10.4141/ cjas88-003
Stahringer, R. C. 2003. La condición corporal en el manejo del rodeo de cría. EEA-INTA Colonia Benítez, Chaco. https:/ / inta.gob.ar/ sites/ default/ files/ script-tmpinta_la_condicin_corporal_en_el_manejo_de_rodeo_ de_pdf 